\title{
Clinical evaluation of transvaginal myomectomy surgery: a retrospective study of 138 cases
}

\author{
Yinghan Chen ${ }^{1}$, Danbo Wang ${ }^{2}$, Fang Ren ${ }^{1}$ \\ 'Shengjing Hospital of China Medical University, Shenyang, China \\ ${ }^{2}$ Cancer Hospital of China Medical University, Shenyang, China
}

\begin{abstract}
Objectives:The aim of this study was to evaluate the safety, feasibility, and effectiveness of transvaginal myomectomy surgery. Material and methods: We conducted a retrospective study in Shengjing Hospital of China Medical University. In all, 138 patients underwent transvaginal myomectomy from March 2009 to March 2019. The perioperative clinical data, such as position and size of myomas, operative duration, blood loss, intraoperative and postoperative complications, and hospitalization time were retrospectively analyzed.

Results: All transvaginal myomectomies were performed without conversion to laparotomy. The mean vaginal operating time was 56.0 ( \pm 17.2 ) minutes. The mean operative estimated blood loss was $89.2( \pm 36.8) \mathrm{mL}$. No significant intraoperative complications occurred. The median time of intestinal function recovery after operation was 1 day (range 1-4 days). The median time of hospital stay was 4 days (range 3-10 days); 12 (8.7\%) patients experienced postoperative morbidity.

Conclusions: Transvaginal myomectomy is a minimally invasive surgery that can be performed without leaving a scar on the body surface. It can be performed safely and effectively by a skilled surgeon in cases with a specific surgical indication for this approach.
\end{abstract}

Key words: uterine myomas; natural orifice surgery; vaginal myomectomy

Ginekologia Polska 2019; 90, 10: 617-621

\section{INTRODUCTION}

Uterine fibroids are the most common benign tumors of the female reproductive organs, being found in $20-30 \%$ of women of reproductive age [1]. Leiomyoma is an important clinical condition in gynecology. It causes abnormal uterine bleeding, pelvic pressure symptoms, spontaneous abortion, and pelvic pain [2]. Most patients require surgical treatment for symptomatic fibroids. Since uterine fibroids are well-lined and non-capsulated benign tumors, myomectomy is an effective treatment option for symptomatic fibroids [3]. In recent years, with increased awareness of health care and greater requirements for quality of life, the incidence of myomectomy is increasing $[4,5]$. In addition to the classical treatment strategy of laparotomy, other techniques such as laparoscopy, operative hysteroscopy and the transvaginal route have been widely used for uterine fibroids in recent decades [6]. The choice of approach for a myomectomy depends on the size, number, and location of fibroids, as well as the skill of the surgeon. In 1994, Magos et al. [7] first reported 3 cases of transvaginal myomectomy. Compared with lapa- roscopy, transvaginal myomectomy is considered to offer many advantages, such as leaving no external scarring, low medical cost, and quick postoperative recovery. Being that it is less invasive than laparoscopy, there is also a better psychological outcome for the patient $[8,9]$. However, due to limited operating space, a restricted field of view, and other relevant complications, the application of transvaginal myomectomy is not as widespread as that of laparoscopic surgery [10]. This paper retrospectively analyzed the clinical data, complications, and outcomes of a series of patients who underwent transvaginal myomectomy performed by experienced surgeons over the past 10 years. The goals of this study are to investigate the safety, feasibility, and effectiveness of this surgical procedure.

\section{MATERIAL AND METHODS Subjects}

This study collected 138 cases of patients with complete medical records, who underwent transvaginal myomectomy in the Department of Obstetrics and Gynecology, Shengjing 


\begin{tabular}{|c|c|}
\hline \multicolumn{2}{|l|}{ Characteristics of patients } \\
\hline Mean age, years $\pm S D$ & $39.6 \pm 11.5$ \\
\hline Nulliparous, n (\%) & $10(7.2)$ \\
\hline Parity, median (range) & $1(0-4)$ \\
\hline \multicolumn{2}{|l|}{ Previous surgery, $\mathrm{n}(\%)$} \\
\hline Previous cesarean section & $13(9.4)$ \\
\hline Oophorocystectomy & $5(3.6)$ \\
\hline Adnexectomy & $2(1.4)$ \\
\hline Tubal ligation & $2(1.4)$ \\
\hline Laparotomic myomectomy & $1(7.2)$ \\
\hline Appendectomy & $3(2.2)$ \\
\hline \multicolumn{2}{|l|}{ Symptomatic uterine fibroids, $\mathrm{n}(\%)$} \\
\hline Menorrhagia & $24(17.4)$ \\
\hline Menostaxis & $12(8.7)$ \\
\hline Frequent micturition Symptoms of bladder compression & $5(3.6)$ \\
\hline Dysmenorrhea & $2(1.4)$ \\
\hline Rapid myoma growth & $3(2.2)$ \\
\hline
\end{tabular}

Hospital of China Medical University from March 2009 to March 2019. The characteristics of the patients are reported in Table 1. All of the patients provided informed consent. This study was approved by the Ethics Committee of our hospital.

\section{Preoperative assessments}

In addition to a complete medical history, all patients underwent careful physical and ultrasound examinations to comprehensively evaluate the uterus and fibroids (size, location, morphology, and activity). Some patients also underwent pelvic magnetic resonance imaging (MRI). Before transvaginal myomectomy, cervical cytology was routinely performed on all patients, and diagnostic curettage was performed on patients with abnormal menstruation to exclude endometrial lesions. The preoperative examinations excluded the ovarian diseases, pelvic adhesions, severe endometriosis, or pelvic inflammation.

\section{Preoperative preparation}

Sexual activity was forbidden for all patients for one week before surgery, and iodophor was used for vaginal disinfection twice per day for three days before the surgery. If vaginal inflammation was present before surgery, transvaginal myomectomy was not performed until the vaginal inflammation was cured. All patients underwent routine preoperative mechanical and chemical bowel preparation and received a single dose of prophylactic intravenous antibiotics immediately before the start of the procedure. The procedure was performed under general endotracheal anesthesia in the lithotomy position.

\section{Surgical procedure}

If uterine fibroids were located in the posterior wall of the uterus, the posterior vaginal wall was incised at the uterosacral ligament to expose the pouch of Douglas and access the retroperitoneum. If uterine fibroids were located in the anterior wall of the uterus, an incision was made along the anterior fornix bilaterally and inwardly until the anterior peritoneum was reached. If the anterior wall space was limited, it was enlarged by an inverted " $\mathrm{T}$ " incision; if the uterus was large, the anterior and posterior fornices were incised simultaneously to enter the abdominal cavity, which is conducive to everting the uterus. After entering the abdomen, the location, size, and number of fibroids were explored. The uterus and uterine fibroids were exposed, and the fibroids were clamped and pulled outward. Pituitrin (6-12 U) was injected into the myometrium in the vicinity of the tumor. The myometrium was bisected to the fibroid pseudocapsule, and uterine fibroids were dissected. If a fibroid was large and could not be completely removed via the vagina, it was separated while being morcellated into wedge-shaped pieces, so it could be removed via the vagina. After the removal of larger fibroids, the whole uterine body was pulled into the vagina. Careful examination was undertaken by the surgeon of the uterine body for small fibroids, which were removed if present. The fibroid pseudocapsule was trimmed using 1-0 absorbable sutures to suture the uterus with intermittent sutures of 1 to 2 layers of the myometrium and continuous suturing of the serosa layer. Subsequently, 2-0 absorbable sutures were used to suture the peritoneal reflection and the vaginal incisal margin, and a T-type tube was placed in the vaginal incision for drainage. The vagina was then filled with two pieces of iodophor-soaked gauze, a ureteral catheter was placed, and the surgery was completed.

\section{Postoperative management and follow-up}

Postoperative routine intravenous infusion of antibiotics was performed for 3 to 5 days. The patients were given a liquid diet for 6 hours after surgery and a semiliquid diet on the following day. The vaginal gauze and ureteral catheter were removed 24 hours post-surgically. Vaginal drainage generally lasted for approximately 48 to 72 hours. Routine monitoring of body temperature, hemography, and exhaust time were performed. At the 2-month, 6-month and 12-month follow-up visits to the general outpatient department, general physical examinations, gynecological examinations, and pelvic ultrasounds were performed.

\section{Observation indicators}

According to the clinical data, the following indicators were collected: the location, number, and volume of uterine fibroids; operative time; intraoperative blood loss; intra- 
operative and postoperative complications; postoperative morbidity; length of postoperative hospital stay; and postoperative fever. Fever was defined as temperature increasing to greater than $38^{\circ} \mathrm{C}$ on two occasions at least 6 hrs apart within 24 hrs, commencing 24 hrs after surgery.

\section{Statistical analysis}

Statistical analysis was performed using SPSS software, version 16 (SPSS Inc. Chicago, IL, USA). The results are expressed as the mean \pm standard deviation.

\section{RESULTS}

\section{Characteristics of uterine fibroids}

The characteristics of uterine fibroids in the 138 cases of transvaginal myomectomy are shown in Table 2. Greater than half of the cases (60.9\%) had multiple fibroids. Of these cases, 8 had greater than 5 fibroids, and the greatest number of fibroids removed in one case was 13 . Most cases $(85.5 \%)$ had fibroids in the uterine body: 12 cases had cervical fibroids, and 8 cases had broad ligament fibroids. In two-thirds of cases (66.7\%), the maximum fibroid diameter was between 5 and $10 \mathrm{~cm}$, and the largest fibroid removed had a diameter of $12 \mathrm{~cm}$.

\section{Perioperative performance data}

All 138 cases were successfully completed, with no adjacent organ injury or other intraoperative complica-

\begin{tabular}{|c|c|}
\hline \multicolumn{2}{|l|}{ Characteristics of Myomas } \\
\hline Single, $n(\%)$ & $54(39.1)$ \\
\hline \multicolumn{2}{|l|}{ Multiple, n (\%) } \\
\hline$\leq 5$ & $76(55.1)$ \\
\hline$>5, \leq 10$ & $6(4.3)$ \\
\hline$>10$ & $2(1.4)$ \\
\hline \multicolumn{2}{|l|}{ Corpus myoma, n (\%) } \\
\hline Subserosal & $47(34.1)$ \\
\hline Intramural & $54(39.1)$ \\
\hline Intramural/Subserosal & $17(12.3)$ \\
\hline submucous & 0 \\
\hline \multicolumn{2}{|l|}{ Special position, n (\%) } \\
\hline Cervical myoma & $11(8.0)$ \\
\hline Cervical/corpus & $1(0.7)$ \\
\hline Broad ligament myoma & $8(5.8)$ \\
\hline Broad ligament/corpus & 0 \\
\hline Myoma size $\mathrm{cm} \pm \mathrm{SD}$ & $6.7 \pm 1.8$ \\
\hline Diameter $\leq 5 \mathrm{~cm}, \mathrm{n}(\%)$ & $43(31.2)$ \\
\hline Diameter $>5 \mathrm{~cm} \leq 10 \mathrm{~cm}, \mathrm{n}(\%)$ & $92(66.7)$ \\
\hline Diameter > $10 \mathrm{~cm}, \mathrm{n}(\%)$ & $3(2.2)$ \\
\hline
\end{tabular}

tions. Perioperative performance data are summarized in Table 3. No postoperative complications occurred. Postoperative analgesia was considered routine for the transvaginal myomectomy procedure. Postoperative pathological examinations revealed uterine fibroids.

Postoperative morbidity: Twelve patients had postoperative temperatures greater than $38^{\circ} \mathrm{C}$, and the postoperative morbidity rate was $8.7 \%$. All patients underwent blood culture and drug susceptibility tests, and Escherichia coli was detected in the cultures of two patients. The symptoms of ten patients resolved 4 to 6 days after combined treatment with antibiotics and traditional Chinese medicines that promote blood circulation and reverse blood stasis. The two other patients developed postoperative uterine and pelvic abscesses, which were treated with interventional ultrasound-guided puncture and pus aspiration. No other complications occurred after antibiotic treatment. All patients with fever were treated with early surgery. The operative time was a minimum of 1.5 hours.

\section{Prognosis}

The mean time to follow-up with patients was $9.0 \pm 2.2$ months (3-12 months). There were 43 patients that underwent surgery due to menometrorrhagia, menostaxis, frequent urination due to bladder compression, or lower abdominal pain. All symptoms were alleviated after surgery; no residual fibroids were found on gynecological examination or ultrasonography. Postoperative follow-up visits showed good healing of the fornix mucosa. Four patients had fertility requirements. Because contraception was required for 2 years, and the follow-up period was short, no postoperative pregnancies have been reported.

\section{DISCUSSION}

Though transvaginal myomectomy has many benefits as mentioned, it is not suitable for all patients who require myomectomy. However, there is currently no uniform standard for indications for transvaginal myomectomy [11]. Some scholars have suggested that the size of the uterus should not exceed the gravid uterus at 12,14 , or 16 weeks

\begin{tabular}{|l|l|}
\hline \multicolumn{2}{|l|}{ Table 3. Perioperative performance } \\
\hline Characteristic & Results \\
\hline Mean operative time (min \pm SD, range) & $56.0 \pm 17.2(30-110)$ \\
\hline Mean blood loss (mL \pm SD, range) & $89.2 \pm 36.8(40-300)$ \\
\hline Blood transfusion rate ( $n, \%)$ & $1(0.7)$ \\
\hline $\begin{array}{l}\text { Mean intestinal function recovery time, } \\
\text { median days (range) }\end{array}$ & $1(1-4)$ \\
\hline $\begin{array}{l}\text { Postoperative fever ( } \mathrm{n}, \%) \\
\text { Mean hospitalization after surgery, } \\
\text { median days (range) }\end{array}$ & $12(8.7)$ \\
\hline
\end{tabular}


of gestation. Others have suggested that the diameter of fibroids should be no more than $7 \mathrm{~cm}, 10 \mathrm{~cm}$ or $11 \mathrm{~cm}$. Still others have suggested that the number of fibroids should not exceed three, while some scholars believe that there should be no restrictions on the number of fibroids. It has also been suggested that fibroids in the anterior wall are not suitable for transvaginal myomectomy [12-15]. We believe that whether transvaginal myomectomy is a suitable treatment option depends on the patient's conditions. The decision to perform transvaginal myomectomy is determined based on a comprehensive assessment of multiple factors, including size, number, type (subserosal, intramural, etc.), and location of fibroids, vaginal conditions, the history of pelvic inflammation, proficiency of the surgeon, and the presence of an adnexal mass. Careful gynecological examination and ultrasonography are very important to determine if the patient is a candidate for this procedure. A MRI should be performed before surgery if necessary. Adequate preoperative assessment and adhering to strict indications are essential for the success of the surgery. Therefore, the incidence of intraoperative and postoperative complications can be avoided or reduced, and the likelihood of conversion to laparotomy can be minimized.

Transvaginal myomectomy has multiple advantages, which have been discussed extensively $[16,17]$. These include advantages in operative time, intraoperative blood loss, degree of postoperative pain, postoperative recovery time of intestinal function, average length of postoperative hospital stay, medical expenses, and aesthetic/psychological effects without abdominal incisions [18]. The results of this study are consistent with the general conclusions of previous studies. We believe that, compared with laparoscopic surgery, the greatest advantage of transvaginal myomectomy is that the surgeon can perform every step of the operation under direct vision, which is more accurate, safe and expedient than under microscopic view. This is true especially when the fibroids are large and buried deeply in the uterus. In the case of a transvaginal approach, conventional layered sutures can be performed on deep uterine muscle wounds, which can improve the reliability of repairing large uterine defects and can compensate for the deficiency of the laparoscopic approach in this area. In addition, because transvaginal myomectomy does not require expensive and complex equipment, it is more suitable for economically underdeveloped areas.

However, transvaginal myomectomy has not been as widely practiced as laparoscopic myomectomy, mainly due to the technical difficulties of transvaginal operations, including limited operating space, restricted field of view and concerns about the injury risk of adjacent organs $[17,19]$.
The key to this procedure is eversion of the uterus; therefore, preoperative examination should determine the location of the major fibroids. If a fibroid protrudes from the anterior wall, it is easy to evert the uterus from the anterior fornix. Otherwise, it should be everted from the posterior fornix. When the volume of the uterus is large, or large fibroids are present, it is often necessary to gradually open the fibroid pseudocapsule while outwardly pulling the uterus. The fibroid is separated until a tenaculum can be used to grasp and remove it. Alternatively, the fibroid can be morcellated while being stripped to reduce the fibroid volume until the fibroid can be fully removed. In addition, the inherently narrow field of vaginal surgery does not have the magnification effect of laparoscopic surgery; thus, vaginal surgery is not conducive to extensive learning and training.

Postoperative fever is a common complication of vaginal myomectomy. Compared with laparoscopic surgery, vaginal myomectomy has a higher postoperative morbidity rate $[11,20]$. In this group of patients, the body temperatures of 12 patients exceeded $38^{\circ} \mathrm{C}$, and two patients developed postoperative uterine and pelvic abscesses, which were relieved by interventional ultrasound-guided puncture and pus aspiration, followed by 4-6 days of combined treatment using antibiotics and traditional Chinese medicines that increase blood circulation and reverse blood stasis. The reason for the high incidence of fever after transvaginal myomectomy is related to the fact that the uterus is a muscular organ with an abundant blood supply [21]. Enucleation of uterine fibroids is a process in which a large number of blood vessels are exposed, and the chance of infection is increased when the uterus, with its exposed blood vessels, remains for a long time in the bacterial environment of the vagina. In this study, all cases of postoperative fever occurred in the early study period. Since preoperative vaginal preparation was emphasized, the occurrence of postoperative fever was significantly reduced after adequate and strict vaginal disinfection. In addition, the operative time of patients with postoperative fever was close to or more than 1.5 hours in this study. With increasing surgical proficiency, the operation time was shortened, thus reducing the uterine exposure time. Therefore, the duration of uterine congestion caused by uterine eversion was minimized, and the occurrence of postoperative fever was also reduced.

In summary, transvaginal myomectomy has its own independent indications and numerous advantages, and it is a useful supplement to the transabdominal operation. As long as the surgeon is proficient in transvaginal surgical skills and is familiar with the surgical indications, it is safe to perform transvaginal myomectomy. 


\section{REFERENCES}

1. De La Cruz MS, Buchanan EM. Uterine Fibroids: Diagnosis and Treatment. Am Fam Physician. 2017; 95(2): 100-107, indexed in Pubmed: 28084714.

2. Donnez J, Dolmans MM. Uterine fibroid management: from the present to the future. Hum Reprod Update. 2016; 22(6): 665-686, doi: 10.1093/humupd/dmw023, indexed in Pubmed: 27466209.

3. Glaser LM, Friedman J, Tsai S, et al. Laparoscopic myomectomy and morcellation: A review of techniques, outcomes, and practice guidelines. Best Pract Res Clin Obstet Gynaecol. 2018; 46: 99-112, doi: 10.1016/j.bpobgyn.2017.09.012, indexed in Pubmed: 29078975.

4. Dubuisson J. The current place of mini-invasive surgery in uterine leiomyoma management. J Gynecol Obstet Hum Reprod. 2019; 48(2): 77-81, doi: 10.1016/j.jogoh.2018.10.004, indexed in Pubmed: 30316905.

5. Wongpia I, Thinkhamrop J, Seejorn K, et al. Incidence of and risk factors for febrile morbidity after laparoscopic-assisted vaginal hysterectomy. Int J Womens Health. 2014; 6: 385-388, doi: 10.2147/IJWH.S57521, indexed in Pubmed: 24748819.

6. Yoong W, Zhao W, Cai H, et al. Vaginal Myomectomy Using the Dührssen (Longitudinal Median Cervical) Incision: A Case Series of $19 \mathrm{~Pa}$ tients. J Minim Invasive Gynecol. 2017; 24(5): 811-814, doi: 10.1016/j. jmig.2017.04.002, indexed in Pubmed: 28411085.

7. Magos AL, Bournas N, Sinha R, et al. Vaginal myomectomy. Br J Obstet Gynaecol. 1994; 101(12): 1092-1094, doi: 10.1111/j.1471-0528.1994. tb13593.x, indexed in Pubmed: 7826970.

8. Rakotomahenina H, Rajaonarison J, Wong L, et al. Myomectomy: technique and current indications. Minerva Ginecol. 2017; 69(4): 357-369, doi: 10.23736/S0026-4784.17.04073-4, indexed in Pubmed: 28447445.

9. Meng L, Li W. The curative effect analysis and nursing measures of the transvaginal myomectomy and transabdominal myomectomy. Pak J Pharm Sci. 2016; 29(6 Suppl): 2281-2285, indexed in Pubmed: 28167466.

10. Flyckt R, Coyne K, Falcone T. Minimally Invasive Myomectomy. Clin Obstet Gynecol. 2017; 60(2): 252-272, doi: 10.1097/GRF.0000000000000275, indexed in Pubmed: 28121646.

11. Flyckt RL. Setting the focus on patient selection for minimally invasive myomectomy: operating times and surgical morbidity in a large database study. Fertil Steril. 2019; 111(6): 1127-1128, doi: 10.1016/j. fertnstert.2019.03.031, indexed in Pubmed: 31043231.
12. Moro F, Bitonti G, Mascilini F, et al. Intraoperative transvaginal ultrasound examination during myomectomy. J Ultrasound. 2019; 22(1): 109-110, doi: 10.1007/s40477-018-0310-9, indexed in Pubmed: 30019287.

13. Chittawar PB, Kamath MS. Review of nonsurgical/minimally invasive treatments and open myomectomy for uterine fibroids. Curr Opin Obstet Gynecol. 2015; 27(6): 391-397, doi: 10.1097/GCO.0000000000000223, indexed in Pubmed: 26536205.

14. Arian SE, Munoz JL, Kim S, et al. Robot-assisted laparoscopic myomectomy: current status. Robot Surg. 2017; 4: 7-18, doi: 10.2147/RSRR. S102743, indexed in Pubmed: 30697559.

15. Lee D, Kim SKi, Kim K, et al. Advantages of Single-Port Laparoscopic Myomectomy Compared with Conventional Laparoscopic Myomectomy: A Randomized Controlled Study. J Minim Invasive Gynecol. 2018 25(1): 124-132, doi: 10.1016/j.jmig.2017.08.651, indexed in Pubmed: 28826957.

16. Rolli R, Favilli A, Acanfora MM, et al. Vaginal myomectomy is a safe and feasible procedure: a retrospective study of 46 cases. J Obstet Gynaecol Res. 2012; 38(9): 1201-1205, doi: 10.1111/j.1447-0756.2012.01852.x, indexed in Pubmed: 22563644.

17. Faivre E, Surroca MM, Deffieux X, et al. Vaginal myomectomy: literature review. J Minim Invasive Gynecol. 2010; 17(2): 154-160, doi: 10.1016/j. jmig.2009.12.007, indexed in Pubmed: 20226401.

18. Rovio PH, Heinonen PK. Transvaginal myomectomy with screw traction by colpotomy. Arch Gynecol Obstet. 2006; 273(4): 211-215, doi: 10.1007/s00404-005-0078-y, indexed in Pubmed: 16208477.

19. Stentz NC, Cooney LG, Sammel MD, et al. Association of Patient Race With Surgical Practice and Perioperative Morbidity After Myomectomy. Obstet Gynecol. 2018; 132(2): 291-297, doi: 10.1097/AOG.0000000000002738, indexed in Pubmed: 29995738.

20. Thubert $T$, Foulot $H$, Vinchant $M$, et al. Surgical treatment: Myomectomy and hysterectomy; Endoscopy: A major advancement. Best Pract Res Clin Obstet Gynaecol. 2016; 34: 104-121, doi: 10.1016/j.bpobgyn.2015.11.021, indexed in Pubmed: 27400649.

21. Wen KC, Horng HC, Wang PH. Hemorrhage: A strong indicator for myomectomy-related complication. J Chin Med Assoc. 2016; 79(8): 413-414, doi: 10.1016/j.jcma.2016.02.004, indexed in Pubmed: 27080090. 